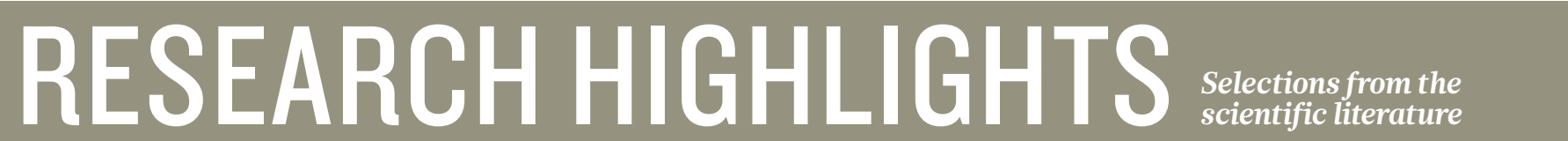

ASTRONOMY

\section{Galaxy from the cosmic dark ages}

Astronomers have found the faintest example yet of a galaxy from the early Universe.

Kuang-Han Huang of the University of California, Davis, and his colleagues spotted the 13-billion-yearold galaxy using the Keck Observatory in Hawaii and the Hubble Space Telescope. A cluster of galaxies in between acted like a lens, gravitationally bending light from the faint galaxy to make it visible to the telescopes.

The detected galaxy is from the end of the 'cosmic dark ages' - when ultraviolet radiation from the earliest stars ionized the Universe's hydrogen to generate the levels seen today. The authors say that studying more galaxies like this one could reveal whether stars did this alone or had help from other sources, such as black holes.

Astrophys. J. Lett. 823, L14 (2016)

\section{METABOLISM}

\section{Growth factor} treats diabetes

Injecting a protein into rodent brains triggers long-term remission of type 2 diabetes.

Certain types of protein called fibroblast growth factors (FGFs) decrease blood glucose levels when they are injected into the bloodstream of animals. To see whether they target the brain, Michael Schwartz of the University of Washington in Seattle and his colleagues injected the brains of rats and mice that had type 2 diabetes with one-tenth of the amount of FGF1 used for bloodstream injections. They found that blood glucose decreased to normal levels 7 days after injection, and

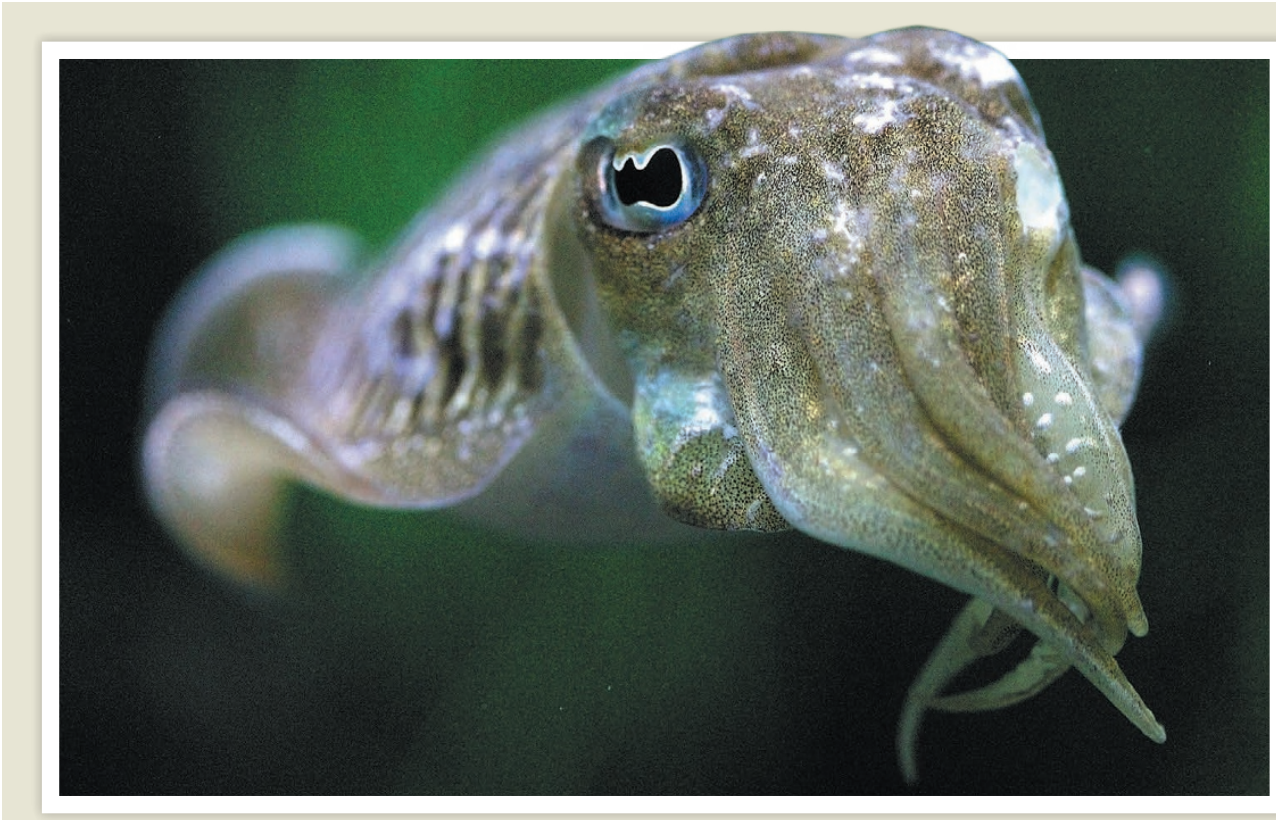

POPULATION ECOLOGY

\section{A boom in octopuses and cuttlefish}

Cephalopods, such as squid, cuttlefish and octopuses, may be benefiting from changes to their environment.

Zoë Doubleday and Bronwyn Gillanders at the University of Adelaide in Australia and their colleagues compiled data from fisheries and scientific marine surveys on global cephalopod catch rates since 1953. They found that cephalopod populations (pictured is a Sepia cuttlefish species) have increased significantly over the past 60 years across some 35 species with different lifestyles, such as ones that live on the sea floor and in the open ocean.

Cephalopods have short lifespans, rapid growth rates and are highly adaptable, which in changing conditions (such as ocean warming) could give them an advantage over slower-growing organisms, the authors say. The cephalopod boom, however, could have damaging effects on their prey populations, such as certain fish and marine invertebrates. Curr. Biol. 26, R406-R407 (2016) stayed that way for up to about 4 months. FGF1 did not change body weight, food intake or blood insulin levels, but glucose was cleared from the circulation into the liver and skeletal muscles twice as fast in treated mice as in untreated ones.

Brain injection of FGF1 may combat diabetes by regulating neural circuits that control how the liver takes up glucose after meals, pointing the way towards possible drug targets, the authors speculate.

Nature Med. http://dx.doi. org/10.1038/nm.4101 (2016)
PLANETARY SCIENCE

\section{End of a Martian ice age}

Frosty layers at Mars's north pole show that the planet is emerging from an ice age.

Mars experiences big climate shifts because of the way it tilts on its axis and orbits the Sun. Isaac Smith of the Southwest Research Institute in Boulder, Colorado, and his colleagues used a radar instrument aboard the Mars Reconnaissance Orbiter spacecraft to hunt for signs of these changes at the north pole. The geometry of the ice layers - sometimes flat, sometimes cutting across one another - allowed the scientists to work out the history of the ice. Some 87,000 cubic kilometres have built up since the end of the last ice age about 370,000 years ago.

The researchers conclude that the ice age is ending because most of this ice accumulated at the north pole, which, unlike on Earth, is warmer than the rest of the planet during an ice age. Science 352, 1075-1078 (2016) 